\title{
Effect of Phoxilium on prognostic predictors in patients undergoing continuous venovenous hemodiafiltration
}

\author{
Da Woon Kim¹, Hyo Jin Kim ${ }^{1,2}$, Jin Mi Kim ${ }^{3}$, You Hyun Jeon ${ }^{1}$, Miyeun Han ${ }^{4}$, Eun Young Seong ${ }^{1,2}$, \\ Sang Heon Song ${ }^{1,2}$ \\ ${ }^{1}$ Department of Internal Medicine, Pusan National University Hospital, Busan, Republic of Korea \\ ${ }^{2}$ Biomedical Research Institute, Pusan National University Hospital, Busan, Republic of Korea \\ ${ }^{3}$ Department of Biostatistics, Clinical Trial Center, Biomedical Research Institute, Pusan National University Hospital, Busan, Republic of Korea \\ ${ }^{4}$ Department of Internal Medicine, Hallym University Hangang Sacred Heart Hospital, Seoul, Republic of Korea
}

Background: Phosphorus-containing dialysis solution is used to prevent hypophosphatemia in patients undergoing continuous venovenous hemodiafiltration (CWHDF). This study evaluated the effect of phosphorus-containing dialysis solution on mortality in patients undergoing CWVHDF based on changes in phosphorus and red cell distribution width-coefficient of variation (RDW-CV) levels.

Methods: We included 272 patients with acute kidney injury (AKI) who underwent CWHDF at the medical intensive care unit from 2017 to 2019 and classified them according to Phoxilium (Baxter Healthcare Ltd.), as a phosphorus-containing dialysis solution, use within 48 hours after CWHDF initiation. Clinical data were collected at baseline and 48 hours after CVHDF initiation. The primary outcome was all-cause mortality during the follow-up period.

Results: The non-Phoxilium (NP) group had higher phosphorus and lower RDW-CV levels than the Phoxilium (P) group (phosphorus, $7.3 \pm 4.3$ vs. $5.0 \pm 2.8 \mathrm{mg} / \mathrm{dL}$; RDW-CV, $14.6 \pm 1.9$ vs. $15.7 \pm 2.6 \%$; all $p<0.001$ ). In the multivariable Cox proportional hazard regression of the NP group, an increase in phosphorus and RDW-CV at 48 hours of CVHDF was associated with mortality (delta phosphorus: median, >0 mg/dL vs. <-2.0 mg/dL; hazard ratio [HR], 8.62; 95\% confidence interval [Cl], 2.10-35.32; $p=0.003 / d e l t a$ RDW-CV: median, >0\% vs. <-0.2\%; HR, 4.34; 95\% Cl, 1.49-13.18; $\mathrm{p}=0.008)$. Meanwhile, in the P group, an increase in delta RDWCV was associated with mortality (delta RDW-CV: >0\% vs. >-0.2\% and <0\%; HR, 2.65; 95\% Cl, 1.12-6.24; $p=0.03$ ), while an increase in delta phosphorus was not.

Conclusion: In patients with AKI undergoing CWHDF, the risk factors for all-cause mortality differed according to the initial phosphorus levels and use of Phoxilium.

Keywords: Continuous renal replacement therapy, Continuous venovenous hemodiafiltration, Phoxilium, Phosphorus, Red cell distribution width

\footnotetext{
Received: November 12, 2020; Revised: April 21, 2021; Accepted: May 11, 2021

Editor: So-Young Lee, CHA University, Seongnam, Republic of Korea

Correspondence: Sang Heon Song

Department of Internal Medicine, Pusan National University Hospital, 179 Gudeok-ro, Seo-gu, Busan 49241, Republic of Korea.

E-mail: shsong0209@gmail.com

ORCID: https://orcid.org/0000-0002-9471-5976
}

Da Woon Kim and Hyo Jin Kim contributed equally to this article as co-first authors.

Copyright (C) 2021 by The Korean Society of Nephrology

(a) This is an Open Access article distributed under the terms of the Creative Commons Attribution Non-Commercial and No Derivatives License (http:// creativecommons.org/licenses/by-nc-nd/4.0/) which permits unrestricted non-commercial use, distribution of the material without any modifications, and reproduction in any medium, provided the original works properly cited. 


\section{Introduction}

Electrolyte imbalances, including that of phosphorus, frequently occur during continuous renal replacement therapy (CRRT) in critically ill patients with acute kidney injury (AKI) [1]. Hypophosphatemia is reported in $11 \%$ to $65 \%$ of patients undergoing CRRT [2]. Severe hypophosphatemia can cause respiratory muscle weakness and decreased cardiac output [2-4]. Hypophosphatemia occurs when phosphorus is removed via CRRT and the intake of phosphorus is reduced. The removal of $57 \mathrm{mmol}$ of phosphorus was observed during a single session of continuous venovenous hemodialysis [5]. Several studies have been conducted to treat hypophosphatemia by replacing phosphorus with dialysate and replacement solutions [6-8]. Thus, Phoxilium (Baxter Healthcare Ltd., Norfolk, UK), a commercially available phosphorus-containing dialysis solution, is now used in patients who require phosphorus supplementation during CRRT.

At present, several prognostic markers for patients on CRRT have been described based on cross-sectional and retrospective studies $[4,9,10]$. Among these, hyperphosphatemia and high red cell distribution width-coefficient of variation (RDW-CV) are predictors of all-cause mortality $[4,9]$. $\mathrm{RDW}-\mathrm{CV}$, expressed as the standard deviation of erythrocyte size divided by the mean corpuscular volume, is a measure of the variation in the red blood cell volumes. In elderly patients with septic shock and an RDW-CV level $>15 \%$, the continuous increase in RDW-CV is a more useful marker for predicting hospital death than the level of RDW-CV itself [11]. In previous studies assessing red cell properties and phosphorus homeostasis, a correlation was observed between CRRT-induced phosphorus depletion and the reduction of RBC 2,3-diphosphoglycerate concentration [2,12-14].

Phoxilium contains phosphorus but less bicarbonate than Hemosol-B0 (Baxter Healthcare Ltd.). In a previous study, after 36 to 42 hours of CRRT, Phoxilium increased serum phosphorus level, while Hemosol-B0 decreased phosphorus level. Additionally, Phoxilium decreased serum bicarbonate level further than Hemosol-B0 [15]. Thus, dialysates and replacement fluids with phosphorus may affect the prognosis of CRRT patients. However, the effect of Phoxilium on prognostic predictors in AKI patients undergoing CRRT has rarely been addressed. Therefore, we aimed to evaluate the effect of Phoxilium use on prognostic predictors for all-cause mortality in AKI patients undergoing CRRT in an intensive care unit
(ICU) in Korea.

\section{Methods}

\section{Study design and subjects}

This was a retrospective single-center study. Data from the medical records of 1,213 patients who underwent CRRT from January 2017 to December 2019 in the ICU of a single-center, university-affiliated hospital were reviewed (Fig. 1). All patients were older than 18 years. The exclusion criteria were as follows: non-internal medicine ICU, history of endstage kidney disease requiring maintenance dialysis, active malignancies, use of Phoxilium 48 hours after CRRT initiation, and insufficient data. Among the 1,213 patients, only 272 were included in the analysis after applying the exclusion criteria. Patients were categorized into two groups according to the use of Phoxilium within 48 hours after CRRT initiation: the non-Phoxilium group (NP group, $\mathrm{n}=96$ ) and the Phoxilium group using Phoxilium as a dialysate or replacement solution or both (P group, $\mathrm{n}=176$ ). The composition of the CRRT fluid is described in Supplementary Table 1 (available online). Phoxilium is the only phosphorus-containing solution among all dialysates and contains $4 \mathrm{mmol} / \mathrm{L}$ potassium. Hemosol-B0 contained neither phosphorus nor potassium. PrismaSol 2 and PrismaSol 4 (Baxter Healthcare Ltd.) contained 2 and $4 \mathrm{mmol} / \mathrm{L}$ potassium, respectively. Phoxilium contains less bicarbonate and calcium than $\mathrm{He}$ mosol-B0 or PrismaSol 2 and PrismaSol 4. The dialysate and replacement solutions were selected based on serum phosphorus and potassium levels according to our hospital protocol (Supplementary Table 2, available online).

The study was approved by the Institutional Review Board of Pusan National University Hospital (No. 2005-005-090), which waived the requirement for informed patient consent because of the retrospective design of the study. All clinical investigations were conducted in accordance with the principles of the Declaration of Helsinki.

\section{Clinical data collection and laboratory measurements}

The demographic and clinical data including age, sex, body mass index (BMI), causes of AKI, and comorbidities at the time of CRRT initiation were reviewed. Laboratory tests were performed in all patients at the time of CRRT initiation and 


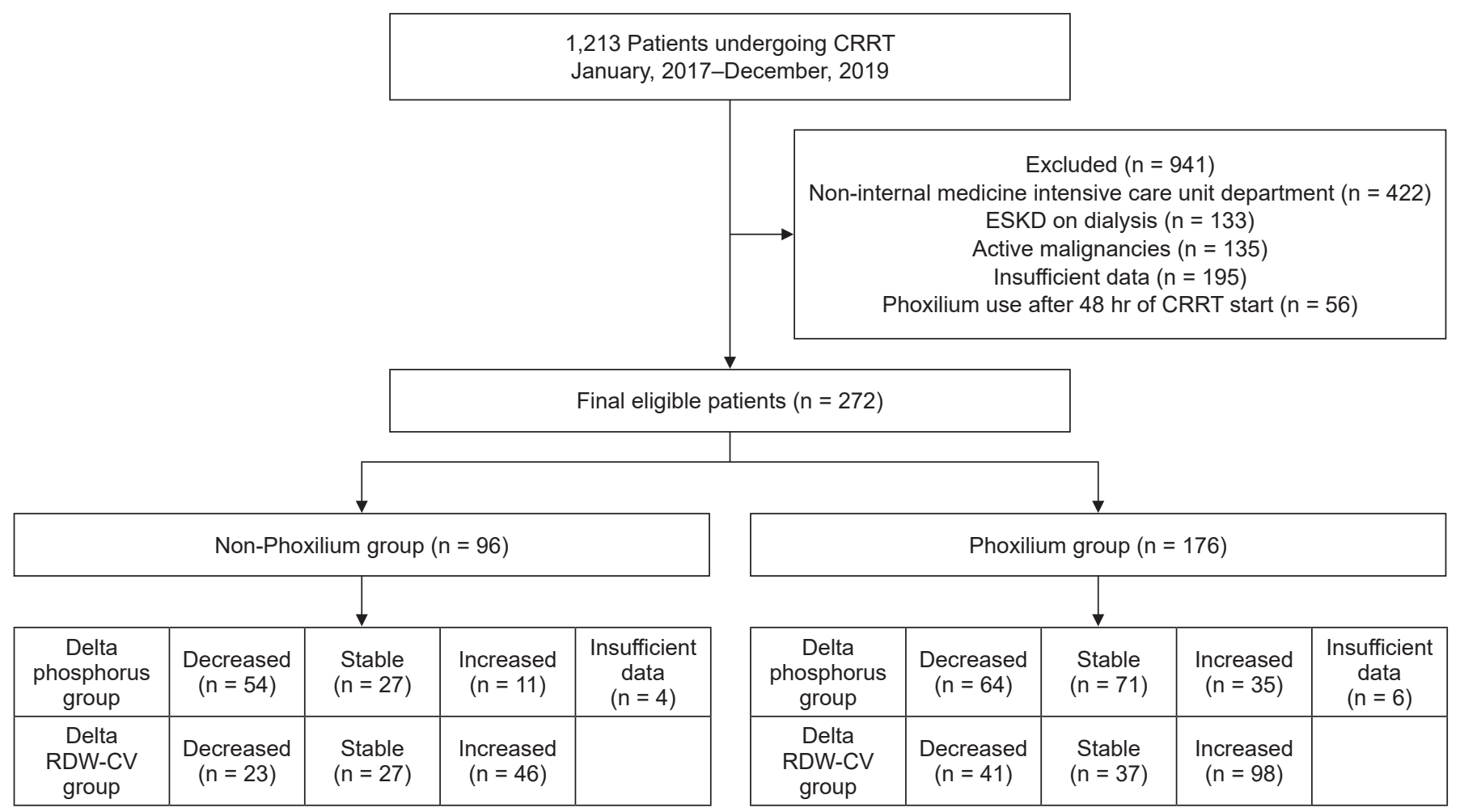

Figure 1. Flow chart of patient selection.

CRRT, continuous renal replacement therapy; ESKD, end-stage kidney disease; RDW-CV, red cell distribution width-coefficient of variation.

48 hours after CRRT initiation. The levels of white blood cells, hemoglobin, RDW-CV, albumin, potassium, bicarbonate, serum blood urea nitrogen, creatinine, phosphorus, calcium, and C-reactive protein (CRP) were measured. The Sequential Organ Failure Assessment (SOFA) score and Acute Physiology and Chronic Health Evaluation II (APACHE II) scores were calculated to assess the severity of the disease [16]. Hyperphosphatemia was defined as a phosphorus level higher than $4.5 \mathrm{mg} / \mathrm{dL}$ and hypophosphatemia as a phosphorus level lower than $2.0 \mathrm{mg} / \mathrm{dL}$.

\section{CRRT protocol}

Among critically ill patients with AKI, patients who had sustained oliguria, uncontrolled volume overload, intractable hyperkalemia, severe metabolic acidosis, or other conditions, including uremic encephalopathy, were subjected to CRRT according to the discretion of their physicians. All patients received continuous venovenous hemodiafiltration
(CVVHDF) through the internal jugular or femoral vein. The Prismaflex (Gambro Lundia AB, Lund, Sweden) CRRT machine and AN 69 ST 100 filter set $\left(1.0 \mathrm{~m}^{2}\right.$, Gambro Lundia $\mathrm{AB})$ were used. The initial effluent flow rates were 30 to 35 $\mathrm{mL} / \mathrm{kg} / \mathrm{hr}$, and additional adjustments were made according to the catabolic state or the presence of hyperkalemia and metabolic acidosis. The actual delivered dose was calculated as the mean value of the effluent volume divided by the weight of the patient during the entire CRRT period. Down time was calculated by adding all the time (hours) for which the CRRT was interrupted during the entire CRRT period. The patients' body weights were measured consecutively during the CRRT period. The blood flow rate was started at $150 \mathrm{~mL} / \mathrm{min}$ and adjusted according to patients' metabolic demands and hemodynamic instabilities. Heparin-free, heparin, or nafamostat mesylate were selected to maintain patency of the extracorporeal circuit while minimizing patient complications according to the bleeding risks of the patients. 


\section{Outcomes}

Patients were followed until February 2020. The primary outcome of the study was all-cause mortality during the follow-up period.

\section{Statistical analysis}

Continuous variables were expressed as the mean \pm standard deviation or median (interquartile range), while categorical variables were expressed as number (percentage). Comparisons between the two groups were performed using Student $t$ test or the Mann-Whitney $U$ test for continuous variables and the chi-square test for categorical variables. Cumulative patient survival curves were derived using the Kaplan-Meier method, and the differences between the curves were analyzed using the log-rank test. The Cox proportional hazards model was used to determine the hazard ratio (HR) of variables related to mortality in the univariable and multivariable analyses. Variables were selected for multivariate analysis according to the study's interest and were serially adjusted. The results are reported as HR and 95\% confidence interval (CI). We applied the same univariable and multivariable Cox proportional hazard regression analyses to variables at 0 and 48 hours of CRRT in the NP and $\mathrm{P}$ groups. Variables in the multivariable Cox regression analysis were sequentially entered into three models. Model 1 was unadjusted. Model 2 was adjusted for age, sex, BMI, phosphorus level, and RDW-CV. In model 3, the SOFA score as well as bicarbonate and albumin levels were added to the covariates in model 2 . We also categorized patients into three groups according to the changes in phosphorus and RDW-CV levels over a 48-hour period after CRRT initiation: delta phosphorus $=$ phosphorus measured at 48 hours (referred to as phosphorus_48hr) - 0 phosphorus measured at $0 \mathrm{hr}$ (referred to as phosphorus_0hr) and delta RDW-CV = RDW-CV at 48 hours (referred to as RDW-CV_48hr) - RDW$\mathrm{CV}$ at $0 \mathrm{hr}$ (referred to as RDW-CV_0hr). Delta phosphorus groups were categorized as increased (delta phosphorus > 0 ), stable (delta phosphorus between median and 0), and decreased (delta phosphorus < median). Similarly, delta RDW-CV groups were categorized as increased (delta RDW$\mathrm{CV}>0$ ), stable (delta RDW-CV between median and 0), and decreased (delta RDW-CV < median). We divided the stable and decreased phosphorus and RDW-CV groups using the median values of the changes at $-2.0 \mathrm{mg} / \mathrm{dL}$ and $-0.2 \%$, respectively. Then, we performed univariable and multivariable Cox proportional hazard regression analysis adjusting for the age, sex, BMI, SOFA score, bicarbonate level, and albumin level at 0 hour. All probabilities were two-tailed, and the level of statistical significance was defined as $p<0.05$. All statistical analyses were performed using IBM SPSS version 20.0 (IBM Corp., Armonk, NY, USA).

\section{Results}

\section{Baseline clinical characteristics of patients}

The baseline clinical characteristics according to Phoxilium use are presented in Table 1. The mean age of patients was

Table 1. Baseline clinical characteristics according to Phoxilium use

\begin{tabular}{|c|c|c|c|c|}
\hline Characteristic & All & Phoxilium (-) & Phoxilium (+) & p-value \\
\hline No. of patients & 272 & 96 & 176 & \\
\hline Age (yr) & $67.0 \pm 13.6$ & $67.4 \pm 12.2$ & $66.7 \pm 14.3$ & 0.67 \\
\hline \multicolumn{5}{|l|}{ Underlying comorbidity } \\
\hline Hypertension & $157(57.7)$ & $62(64.6)$ & $95(54.0)$ & 0.09 \\
\hline Diabetes mellitus & $141(51.8)$ & $53(55.2)$ & $88(50.0)$ & 0.41 \\
\hline Liver disease & 39 (14.3) & $11(11.5)$ & $28(15.9)$ & 0.32 \\
\hline Body mass index $\left(\mathrm{kg} / \mathrm{m}^{2}\right)$ & $23.3 \pm 4.3$ & $23.0 \pm 4.2$ & $23.5 \pm 4.4$ & 0.36 \\
\hline Systolic blood pressure (mmHg) & $113.4 \pm 24.5$ & $111.9 \pm 18.9$ & $114.2 \pm 27.1$ & 0.42 \\
\hline Diastolic blood pressure (mmHg) & $65.5 \pm 15.1$ & $66.7 \pm 12.9$ & $64.9 \pm 16.2$ & 0.33 \\
\hline
\end{tabular}


Table 1. Continued

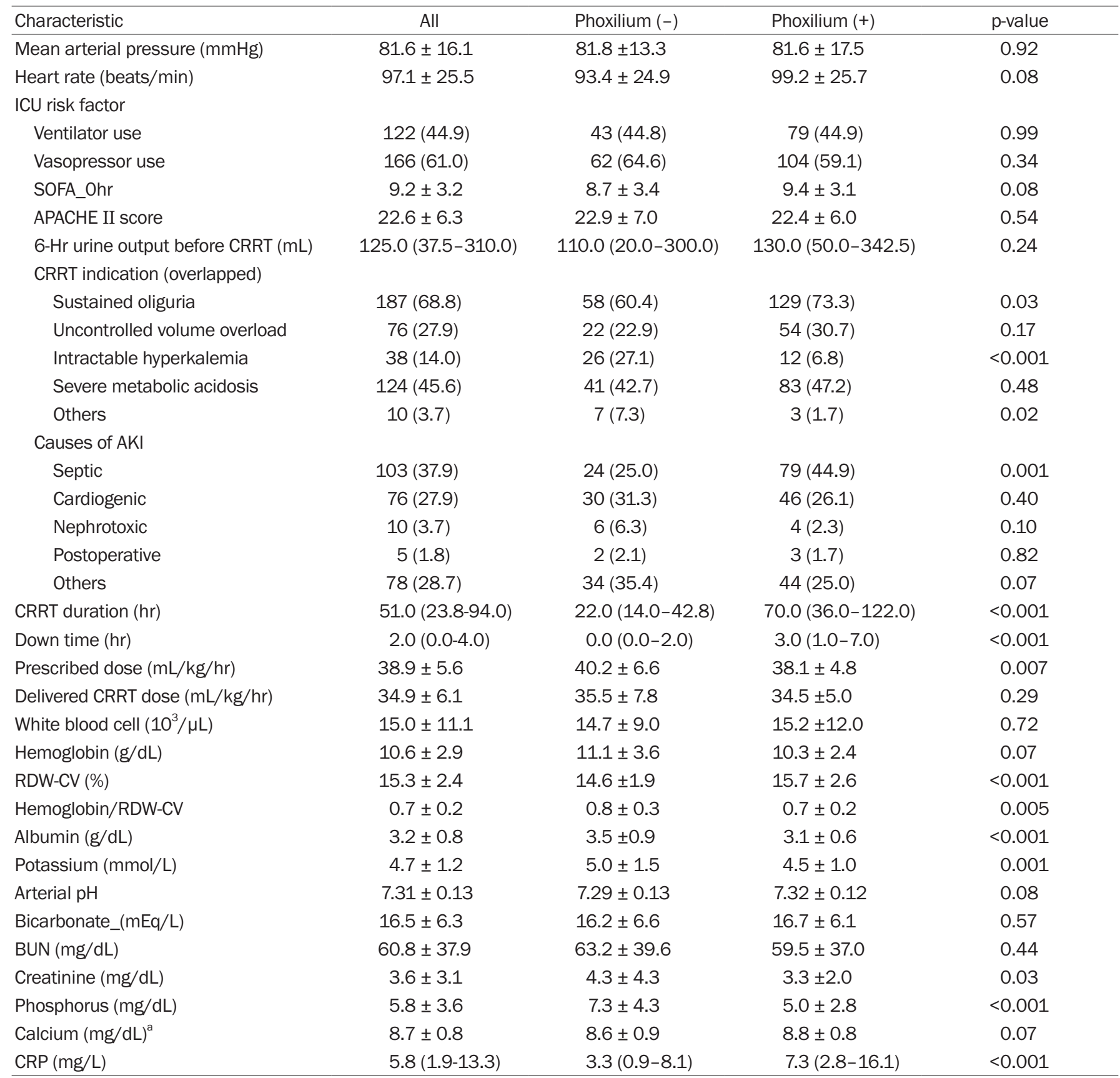

Data are expressed as number only, mean \pm standard deviation, number (\%), or median (interquartile range).

Phoxilium (-): The group of patients who did not receive Phoxilium (Baxter Healthcare Ltd., Norfolk, UK) within 48 hours after CRRT initiation. Phoxilium (+): The group of patients who received Phoxilium within 48 hours after CRRT initiation. Down time: duration of unplanned interruption of CRRT therapy during which patients could not receive dialysis.

AKI, acute kidney injury; APACHE II, Acute Physiology And Chronic Health Evaluation II; BUN, blood urea nitrogen; CRP, C-reactive protein; CRRT: continuous renal replacement therapy; ICU, intensive care unit; RDW-CV, red cell distribution width-coefficient of variation; SOFA, Sequential Organ Failure Assessment. ${ }^{\mathrm{a}}$ Corrected calcium $(\mathrm{mg} / \mathrm{dL})=$ measured total calcium $(\mathrm{mg} / \mathrm{dL})+0.8 \times[4-$ measured serum albumin $(\mathrm{g} / \mathrm{dL})]$.

$67.0 \pm 13.6$ years, and 161 patients $(59.2 \%)$ were males. The mean arterial pressure was $81.6 \pm 16.1 \mathrm{mmHg}$. A total of 122 patients (44.9\%) received mechanical ventilation. The mean SOFA score and APACHE II score were $9.2 \pm 3.2$ and 22.6 
\pm 6.3 , respectively. Urine output for 6 hours before CRRT initiation was $125.0 \mathrm{~mL}(37.5-310.0 \mathrm{~mL})$. The delivered CRRT dose was $34.9 \pm 6.1 \mathrm{~mL} / \mathrm{kg} / \mathrm{hr}$. Comparing the NP and $P$ groups, no significant differences were observed in the baseline characteristics of age, sex, BMI, SOFA_0hr score, or bicarbonate_0hr (all p > 0.05). In the P group, CRRT duration and down time were longer, RDW-CV_Ohr and CRP_0hr were higher, and albumin_0hr and phosphorus_0hr were lower than in the NP group (all p < 0.001). Sepsis was the most common cause of AKI in P group, whereas other causes, including hypovolemic shock and hepatorenal syndrome, were most common in the NP group.

Clinical characteristics at 48 hours after continuous renal replacement therapy initiation according to Phoxilium use

Clinical characteristics at 48 hours after CRRT initiation according to Phoxilium use are presented in Supplementary Table 3 (available online). The SOFA_48hr score was higher $(\mathrm{p}=0.001)$ and bicarbonate_48hr was lower $(\mathrm{p}=0.03)$ in the $P$ group than in the NP group. RDW-CV_48hr was higher in the $\mathrm{P}$ group ( $\mathrm{p}=0.005)$. Phosphorus_48hr decreased to 3.6 $\pm 2.0 \mathrm{mg} / \mathrm{dL}$ in the NP group and to $3.1 \pm 1.1 \mathrm{mg} / \mathrm{dL}$ in the $\mathrm{P}$ group. Hyperphosphatemia was found in 10 patients in the NP group (10.4\%) and 21 patients in the P group (11.9\%) at 48 hours after CRRT initiation.

\section{All-cause mortality according to baseline clinical pa- rameters}

The mean follow-up duration was 15.0 days (7.0-25.8 days) in the NP group vs. 24.0 days (14.0-38.0 days) in the P group. During the follow-up period, 94 patients (34.1\%) died, including 26 (27.1\%) from the NP group and 68 (38.6\%) from the P group (Supplementary Table 4, available online). The Cox regression analysis results for all-cause mortality according to the baseline clinical parameters are presented in Table 2. The use of Phoxilium within 48 hours after CRRT initiation was not a risk factor for all-cause mortality ( $\mathrm{p}=$ 0.76). In addition, the additional analysis of Kaplan-Meier curves between the groups of patients using Phoxilium as dialysate solutions $(n=45)$, replacement solutions $(n=91)$, and both $(n=40)$ showed that time to death was not different ( $\log$-rank $\mathrm{p}=0.73$ ). In the NP group, SOFA_0hr (HR, 1.38; 95\% CI, 1.20-1.59; p < 0.001), bicarbonate_0hr (HR, 1.08; 95\%
CI, 1.02-1.15; $\mathrm{p}=0.007$ ), and albumin_0hr (HR, 0.50; 95\% CI, 0.29-0.86; $p=0.01$ ) were associated with increased all-cause mortality in model 1. RDW-CV_0hr was not a significant risk factor for all-cause mortality. In the fully adjusted model 3, higher SOFA_0hr (HR, 1.38; 95\% CI, 1.16-1.64; p < 0.001) and higher bicarbonate_0hr (HR, 1.08; 95\% CI, 1.00-1.17; p $=0.05$ ) were still significantly associated with increased allcause mortality.

In the P group, RDW-CV_Ohr (HR, 1.16; 95\% CI, 1.06-1.26; $\mathrm{p}=0.001)$, SOFA_0hr (HR, 1.13; 95\% CI, 1.05-1.22; $\mathrm{p}=0.001)$, bicarbonate_0hr (HR, 1.04; 95\% CI, 1.00-1.08; $\mathrm{p}=0.05$ ), and albumin_0hr (HR, 0.52; 95\% CI, 0.33-0.81; p = 0.004) were associated with increased all-cause mortality in model 1. Phosphorus_0hr was not a significant risk factor for all-cause mortality. In the fully adjusted model 3, higher SOFA_0hr (HR, 1.14; 95\% CI, 1.04-1.26; $\mathrm{p}=0.005$ ), higher bicarbonate_0hr (HR, 1.05; 95\% CI, 1.01-1.09; p = 0.03), and lower albumin_0hr (HR, 0.51; 95\% CI, 0.29-0.89; $\mathrm{p}=0.02$ ) were significant risk factors for all-cause mortality, but RDW-CV_0hr was not.

All-cause mortality according to clinical parameters at 48 hours after continuous renal replacement therapy initiation

The Cox regression analysis results for all-cause mortality according to the clinical parameters at 48 hours are presented in Table 3. In the NP group, SOFA_48hr (HR, 1.31; 95\% CI, 1.12-1.53; $\mathrm{p}=0.001$ ) was the only risk factor for all-cause mortality in the fully adjusted model 3 . In the P group, higher RDW-CV_48hr (HR, 1.15; 95\% CI, 1.04-1.28; $\mathrm{p}=0.008$ ), phosphorus_48hr (HR, 1.41; 95\% CI, 1.09-1.82; p = 0.009), and SOFA_48hr (HR, 1.11; 95\% CI, 1.03-1.20; p = 0.009) and lower bicarbonate_48hr (HR, 0.92; 95\% CI, 0.85-1.00; p = $0.05)$ were associated with increased all-cause mortality in the fully adjusted model 3 .

All-cause mortality according to changes in clinical parameters during 48 hours of continuous renal replacement therapy

Changes in phosphorus and RDW-CV levels during 48 hours of CRRT were evaluated as risk factors for all-cause mortality. As mentioned above, we divided the patients into three groups according to the changes in phosphorus and 
Table 2. All-cause mortality according to Phoxilium use (initial clinical characteristics)

\begin{tabular}{|c|c|c|c|c|c|c|c|c|c|}
\hline \multirow{2}{*}{ Variable } & \multicolumn{3}{|c|}{ Model 1} & \multicolumn{3}{|c|}{ Model 2} & \multicolumn{3}{|c|}{ Model 3} \\
\hline & $\mathrm{HR}$ & $95 \% \mathrm{Cl}$ & p-value & $\mathrm{HR}$ & $95 \% \mathrm{Cl}$ & p-value & HR & $95 \% \mathrm{Cl}$ & $p$-value \\
\hline \multicolumn{10}{|l|}{$\overline{\text { All }}$} \\
\hline Age & 1.00 & $0.99-1.03$ & 0.25 & 1.01 & $0.99-1.03$ & 0.26 & 1.02 & $1.00-1.04$ & 0.06 \\
\hline Male sex & 1.13 & $0.75-1.70$ & 0.57 & 1.02 & $0.65-1.59$ & 0.94 & 1.35 & $0.85-2.14$ & 0.20 \\
\hline $\mathrm{BMI}$ & 0.96 & 0.91-1.01 & 0.13 & 0.96 & $0.91-1.01$ & 0.14 & 0.96 & $0.90-1.01$ & 0.13 \\
\hline Phoxilium use & 1.07 & $0.68-1.69$ & 0.76 & & & & & & \\
\hline RDW-CV & 1.15 & $1.06-1.24$ & $<0.001$ & 1.14 & $1.06-1.23$ & 0.001 & 1.06 & $0.97-1.16$ & 0.22 \\
\hline Phosphorus & 0.95 & $0.88-1.02$ & 0.14 & 0.95 & $0.88-1.03$ & 0.19 & 1.04 & $0.95-1.13$ & 0.43 \\
\hline SOFA & 1.19 & $1.12-1.27$ & $<0.001$ & - & - & - & 1.17 & $1.09-1.27$ & $<0.001$ \\
\hline Bicarbonate & 1.05 & $1.02-1.09$ & 0.003 & - & - & - & 1.05 & $1.02-1.09$ & 0.005 \\
\hline Albumin & 0.53 & $0.74-0.98$ & $<0.001$ & - & - & - & 0.56 & $0.67-0.87$ & 0.009 \\
\hline \multicolumn{10}{|l|}{ Phoxilium (-) } \\
\hline Age & 1.02 & $0.98-1.06$ & 0.35 & 1.00 & $0.96-1.04$ & 0.93 & 1.00 & $0.95-1.04$ & 0.86 \\
\hline Male sex & 0.80 & $0.35-1.84$ & 0.60 & 0.78 & $0.33-1.86$ & 0.57 & 1.28 & $0.48-3.39$ & 0.63 \\
\hline $\mathrm{BMI}$ & 0.97 & $0.88-1.07$ & 0.54 & 0.97 & $0.87-1.07$ & 0.49 & 0.99 & $0.89-1.09$ & 0.79 \\
\hline RDW-CV & 1.11 & $0.92-1.35$ & 0.26 & 1.08 & $0.88-1.32$ & 0.46 & 0.96 & $0.75-1.24$ & 0.77 \\
\hline Phosphorus & 0.81 & $0.69-0.96$ & 0.02 & 0.82 & $0.70-0.97$ & 0.02 & 0.90 & $0.73-1.10$ & 0.29 \\
\hline SOFA & 1.38 & $1.20-1.59$ & $<0.001$ & - & - & - & 1.38 & $1.16-1.64$ & $<0.001$ \\
\hline Bicarbonate & 1.08 & $1.02-1.15$ & 0.007 & - & - & - & 1.08 & $1.00-1.17$ & 0.05 \\
\hline Albumin & 0.50 & $0.29-0.86$ & 0.01 & - & - & - & 0.74 & $0.36-1.56$ & 0.43 \\
\hline \multicolumn{10}{|l|}{ Phoxilium (+) } \\
\hline Age & 1.01 & $0.99-1.03$ & 0.44 & 1.01 & $0.99-1.03$ & 0.50 & 1.02 & $0.99-1.04$ & 0.15 \\
\hline Male sex & 1.28 & $0.79-2.06$ & 0.32 & 1.18 & $0.69-2.00$ & 0.55 & 1.50 & $0.86-2.60$ & 0.15 \\
\hline $\mathrm{BMI}$ & 0.96 & $0.90-1.02$ & 0.15 & 0.96 & $0.90-1.02$ & 0.20 & 0.95 & $0.89-1.02$ & 0.18 \\
\hline RDW-CV & 1.16 & $1.06-1.26$ & 0.00 & 1.15 & $1.05-1.26$ & 0.002 & 1.08 & $0.97-1.19$ & 0.18 \\
\hline Phosphorus & 1.02 & $0.93-1.11$ & 0.71 & 1.01 & $0.91-1.10$ & 0.91 & 1.11 & $1.00-1.23$ & 0.05 \\
\hline SOFA & 1.13 & $1.05-1.22$ & 0.001 & - & - & - & 1.14 & $1.04-1.26$ & 0.005 \\
\hline Bicarbonate & 1.04 & $1.00-1.08$ & 0.05 & - & - & - & 1.05 & $1.01-1.09$ & 0.03 \\
\hline Albumin & 0.52 & $0.33-0.81$ & 0.004 & - & - & - & 0.51 & $0.29-0.89$ & 0.02 \\
\hline
\end{tabular}

Model 1: unadjusted; model 2: age, sex, BMI at intensive care unit admission, and RDW-CV and phosphorus at CRRT initiation; model 3: model 2 + SOFA score, bicarbonate, and albumin at CRRT initiation. Phoxilium (-): the group of patients who did not receive Phoxilium (Baxter Healthcare Ltd., Norfolk, UK) within 48 hours after CRRT initiation. Phoxilium (+): the group of patients who received Phoxilium within 48 hours after CRRT initiation.

$\mathrm{BMI}$, body mass index; Cl, confidence interval; CRRT, continuous renal replacement therapy; HR, hazard ratio; RDW-CV, red cell distribution width-coefficient of variation; SOFA, Sequential Organ Failure Assessment.

RDW-CV levels and eventually chose the lowest mortality group as a reference group. In the NP group composed of 96 patients, the numbers of patients in the delta phosphorus groups were 54 (decreased group), 27 (stable group), and 11 (increased group), while those in the delta RDW-CV groups were 23 (decreased group), 27 (stable group), and 46 (increased group) (Fig. 1). The Kaplan-Meier curve showed that the time to death was significantly different in patients with changes in phosphorus (Fig. $2 \mathrm{~A}, \mathrm{p}<0.001$ ) and RDW$\mathrm{CV}$ levels (Fig. 2B, $\mathrm{p}=0.009$ ). In the Cox regression model after full adjustment, the patients in the increased phos- phorus group showed an 8.62-fold (95\% CI, 2.10-35.32; $\mathrm{p}=$ 0.003 ) increased risk of all-cause mortality compared with the decreased phosphorus group (Table 4). Additionally, patients in the increased RDW-CV group (HR, 4.34; 95\% CI, 1.49-13.18; $\mathrm{p}=0.008$ ) showed increased all-cause mortality compared with the decreased RDW-CV group.

In the P group composed of 176 patients, the numbers of patients in the delta phosphorus groups were 64 (decreased group), 71 (stable group), and 35 (increased group), while those in the delta RDW-CV groups were 41 (decreased group), 37 (stable group), and 98 (increased group) (Fig. 1). 
Table 3. All-cause mortality according to Phoxilium use (48-hr clinical characteristics)

\begin{tabular}{|c|c|c|c|c|c|c|c|c|c|}
\hline \multirow{2}{*}{ Variable } & \multicolumn{3}{|c|}{ Model 1} & \multicolumn{3}{|c|}{ Model 2} & \multicolumn{3}{|c|}{ Model 3} \\
\hline & $\mathrm{HR}$ & $95 \% \mathrm{Cl}$ & $p$-value & HR & $95 \% \mathrm{Cl}$ & p-value & HR & $95 \% \mathrm{Cl}$ & $p$-value \\
\hline \multicolumn{10}{|l|}{ All } \\
\hline Male sex & 1.13 & $0.75-1.70$ & 0.57 & 1.26 & $0.79-2.02$ & 0.34 & 1.20 & $0.71-2.01$ & 0.49 \\
\hline $\mathrm{BMI}$ & 0.96 & $0.91-1.01$ & 0.13 & 0.95 & $0.89-1.00$ & 0.047 & 0.93 & $0.88-0.98$ & 0.01 \\
\hline RDW-CV & 1.19 & $1.11-1.29$ & $<0.001$ & 1.22 & $1.13-1.32$ & $<0.001$ & 1.13 & $1.03-1.24$ & 0.009 \\
\hline SOFA & 1.21 & $1.14-1.27$ & $<0.001$ & - & - & - & 1.13 & $1.03-1.21$ & $<0.001$ \\
\hline Bicarbonate & 0.90 & $0.85-0.95$ & $<0.001$ & - & - & - & 0.96 & 0.90-1.01 & 0.13 \\
\hline Albumin & 0.65 & $0.41-1.01$ & 0.06 & - & - & - & 0.86 & $0.52-1.42$ & 0.55 \\
\hline \multicolumn{10}{|l|}{ Phoxilium (-) } \\
\hline Age & 1.02 & $0.98-1.06$ & 0.35 & 1.02 & $0.98-1.06$ & 0.46 & 1.03 & 0.99-1.08 & 0.19 \\
\hline Phosphorus & 1.37 & $1.17-1.61$ & $<0.001$ & 1.47 & $1.22-1.76$ & $<0.001$ & 1.20 & $0.94-1.54$ & 0.15 \\
\hline SOFA & 1.33 & $1.20-1.47$ & $<0.001$ & - & - & - & 1.31 & $1.12-1.53$ & 0.001 \\
\hline Bicarbonate & 0.90 & $0.83-0.97$ & 0.007 & - & - & - & 0.98 & 0.91-1.06 & 0.63 \\
\hline Albumin & 0.31 & $0.13-0.73$ & 0.007 & - & - & - & 0.73 & $0.25-2.08$ & 0.55 \\
\hline \multicolumn{10}{|l|}{ Phoxilium (+) } \\
\hline Age & 1.01 & $0.99-1.03$ & 0.44 & 1.01 & $0.99-1.03$ & 0.31 & 1.01 & $0.98-1.03$ & 0.53 \\
\hline Male sex & 1.28 & $0.79-2.06$ & 0.32 & 1.34 & $0.76-2.34$ & 0.31 & 1.27 & $0.69-2.36$ & 0.44 \\
\hline BMI & 0.96 & $0.90-1.02$ & 0.15 & 0.95 & $0.89-1.02$ & 0.14 & 0.94 & $0.88-1.01$ & 0.10 \\
\hline RDW-CV & 1.19 & $1.11-1.29$ & $<0.001$ & 1.20 & $1.10-1.32$ & $<0.001$ & 1.15 & $1.04-1.28$ & 0.008 \\
\hline Phosphorus & 1.43 & $1.14-1.79$ & 0.002 & 1.48 & $1.19-1.85$ & 0.001 & 1.41 & $1.09-1.82$ & 0.009 \\
\hline
\end{tabular}

Model 1: unadjusted; model 2: age, sex, BMl at intensive care unit admission, and RDW-CV and phosphorus at CRRT initiation; model 3: model 2 + SOFA score, bicarbonate, and albumin at CRRT initiation. Phoxilium (-): the group of patients who did not receive Phoxilium (Baxter Healthcare Ltd., Norfolk, UK) within 48 hours after CRRT initiation. Phoxilium (+): the group of patients who received Phoxilium within 48 hours after CRRT initiation.

$\mathrm{BMI}$, body mass index; Cl, confidence interval; CRRT, continuous renal replacement therapy; HR, hazard ratio; RDW-CV, red cell distribution width-coefficient of variation; SOFA, Sequential Organ Failure Assessment.

The Kaplan-Meier curve showed that time to death was insignificant with respect to changes in phosphorus levels (Fig. 3A, $\mathrm{p}=0.56$ ) but was significantly affected by changes in RDW-CV levels (Fig. 3B, $\mathrm{p}=0.02$ ). In the Cox regression model after full adjustment, all-cause mortality did not differ according to changes in phosphorus levels (Table 4, all $\mathrm{p}>0.05$ ). Additionally, the increased RDW-CV group (HR, 2.65; 95\% CI, 1.12-6.24; $\mathrm{p}=0.03$ ) and the decreased RDW$\mathrm{CV}$ group (HR, 2.83; 95\% CI, 1.16-6.91; $\mathrm{p}=0.02$ ) showed increased all-cause mortality compared with the stable RDWCV group.

\section{Discussion}

CRRT is an important treatment modality for AKI in critically ill patients. The overall use of CRRT has increased over time, and the proportion of CRRT patients among all acute renal replacement therapy patients reached $80 \%$ after 2014 in Korea, which is relatively higher than that seen in other nations [17-19]. In recent studies, the all-cause mortality and renal survival rates greatly improved after CRRT initiation $[17,18]$. CRRT contributes to the correction of imbalances in electrolytes and mineral parameters as well as acid-base imbalances. However, significant problems of overcorrection 


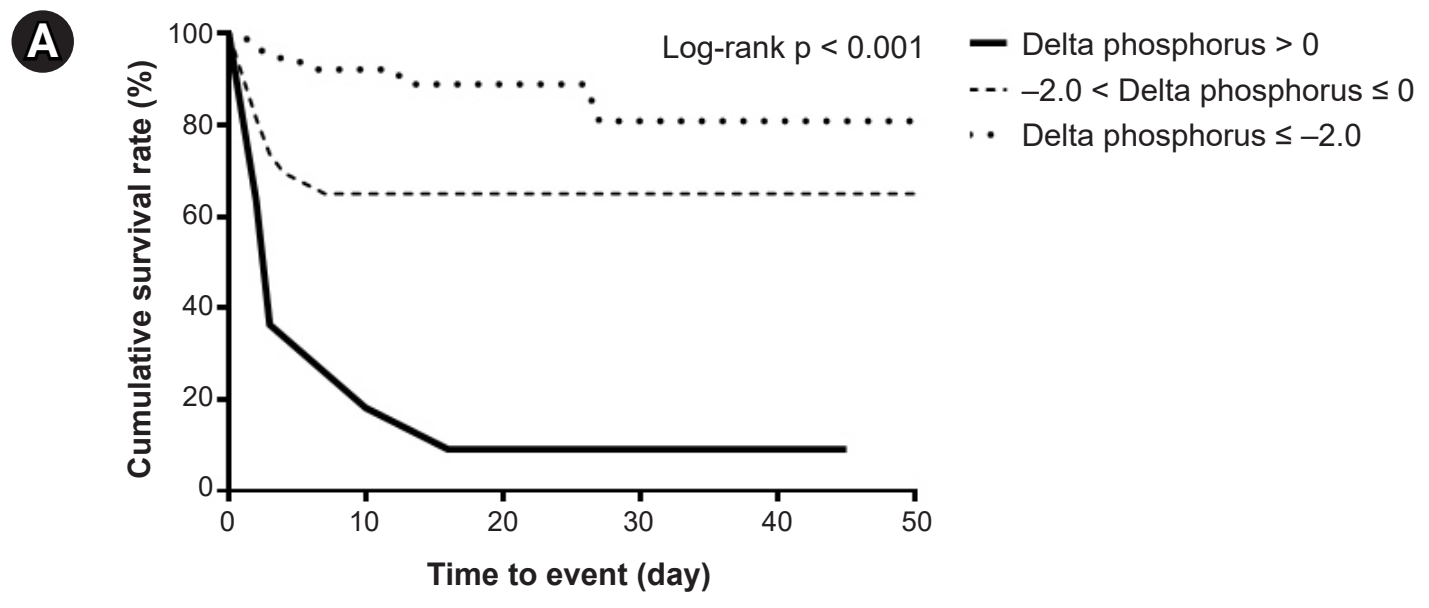

(B)

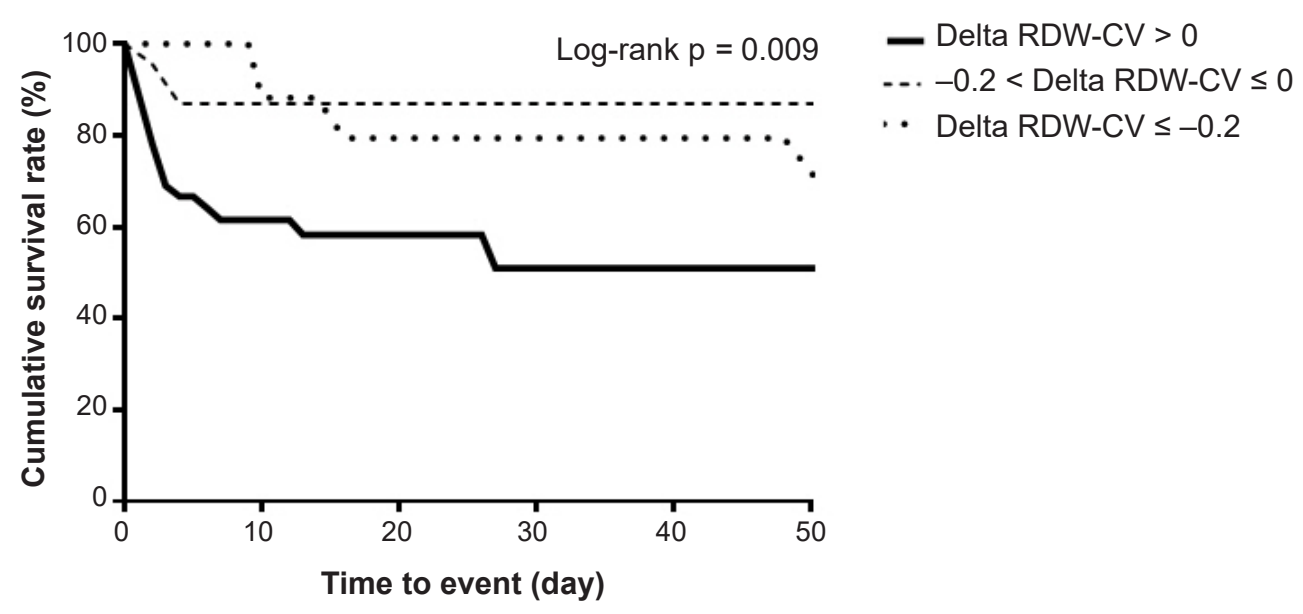

Figure 2. Kaplan-Meier plots for all-cause mortality according to changes in phosphorus and RDW-CV levels during 48 hours of CRRT in the non-Phoxilium group. We categorized patients into three groups according to the changes in phosphorus and RDW-CV levels between 0 hour and 48 hours: increased, stable, and decreased phosphorus and RDW-CV levels. We divided stable and decreased phosphorus and RDW-CV groups using the median value of the changes, $-2.0 \mathrm{mg} / \mathrm{dL}$ and $-0.2 \%$, respectively. In the non-Phoxilium group, the Kaplan-Meier curve for all-cause mortality showed that time to death was significantly different in patients with changes in phosphorus (Fig. 2A, p < 0.001) and RDW-CV levels (Fig. 2B, p=0.009).

CRRT, continuous renal replacement therapy; delta phosphorus, changes in phosphorus during 48 hours of CRRT; delta RDW-CV, changes in RDW-CV during 48 hours of CRRT; RDW-CV, red cell distribution width-coefficient of variation. Phoxilium: Baxter Healthcare Ltd., Norfolk, UK.

or suboptimal parameter correction are still observed [20]. Phoxilium, developed to prevent hypophosphatemia during CRRT, has been proven effective and is used contemporarily [15]. The present study retrospectively analyzed the risk factors for all-cause mortality in groups divided according to Phoxilium use and showed that the baseline characteristics and risk factors of all-cause mortality differed by the initial phosphorus levels and the use of Phoxilium.

In patients with AKI treated with CRRT, higher RDW-CV and CRP levels and lower albumin levels are generally as- sociated with all-cause mortality, presenting with severe inflammation and malnutrition $[9,10]$. However, in our study, Kaplan-Meier analysis results for all-cause mortality showed that CRP_Ohr was not a risk factor for all-cause mortality in all groups combined $(p=0.99)$, the NP group ( $p=0.995)$, or the $P$ group $(p=0.24)$. This result might be due to AKI stemming from variable causes other than sepsis. In the P group, RDW-CV_Ohr and CRP_Ohr were higher, CRRT duration was longer, and albumin_0hr was lower than those in the NP group (all $\mathrm{p}<0.001$ ). Although the mean SOFA_0hr score 
Table 4. All-cause mortality according to delta phosphorus and delta RDW-CV

\begin{tabular}{|c|c|c|c|c|c|c|}
\hline \multirow{2}{*}{ Variable } & \multicolumn{3}{|c|}{ Unadjusted } & \multicolumn{3}{|c|}{ Adjusted } \\
\hline & HR & $95 \% \mathrm{Cl}$ & p-value & HR & $95 \% \mathrm{Cl}$ & p-value \\
\hline \multicolumn{7}{|l|}{ Phoxilium (-) } \\
\hline Delta P group & & & $<0.001$ & & & 0.01 \\
\hline Decreased & Reference & & & Reference & & \\
\hline Stable & 2.93 & $1.09-7.90$ & 0.03 & 2.68 & $0.86-9.33$ & 0.09 \\
\hline Increased & 10.55 & $3.95-28.00$ & $<0.001$ & 8.62 & 2.10-35.32 & 0.003 \\
\hline Delta RDW-CV group & & & 0.02 & & & 0.13 \\
\hline Decreased & Reference & & & Reference & & \\
\hline Stable & 1.17 & $0.26-5.23$ & 0.84 & 1.66 & $0.31-9.04$ & 0.56 \\
\hline Increased & 3.78 & $1.12-12.79$ & 0.03 & 4.34 & $1.49-13.18$ & 0.008 \\
\hline Age & 1.02 & $0.98-1.06$ & 0.35 & 0.99 & $0.95-1.04$ & 0.71 \\
\hline Male sex & 0.80 & $0.35-1.84$ & 0.60 & 2.97 & $0.94-9.44$ & 0.07 \\
\hline $\mathrm{BMI}$ & 0.97 & $0.88-1.07$ & 0.54 & 0.94 & $0.84-1.05$ & 0.26 \\
\hline SOFA & 1.11 & $1.03-1.21$ & 0.007 & 1.24 & $1.05-1.47$ & 0.01 \\
\hline Bicarbonate_Ohr & 0.96 & $0.92-1.00$ & 0.05 & 1.05 & $0.97-1.13$ & 0.24 \\
\hline Albumin_Ohr & 1.70 & $1.04-2.78$ & 0.04 & 0.78 & $0.39-1.55$ & 0.48 \\
\hline \multicolumn{7}{|l|}{ Phoxilium (+) } \\
\hline Delta P group & & & 0.57 & & & 0.41 \\
\hline Decreased & Reference & & & Reference & & \\
\hline Stable & 0.77 & $0.44-1.34$ & 0.36 & 0.68 & $0.39-1.20$ & 0.18 \\
\hline Increased & 1.03 & $0.53-1.98$ & 0.94 & 0.81 & $0.41-1.61$ & 0.55 \\
\hline Delta RDW-CV group & & & 0.03 & & & 0.06 \\
\hline Decreased & 3.09 & $1.31-7.33$ & 0.01 & 2.83 & $1.16-6.91$ & 0.02 \\
\hline Stable & Reference & & & Reference & & \\
\hline Increased & 2.75 & $1.22-6.20$ & 0.02 & 2.65 & $1.12-6.24$ & 0.03 \\
\hline Age & 1.01 & $0.99-1.03$ & 0.44 & 1.01 & $0.99-1.03$ & 0.26 \\
\hline Male sex & 1.28 & $0.79-2.06$ & 0.32 & 1.64 & $0.95-2.86$ & 0.08 \\
\hline BMI & 0.96 & $0.90-1.02$ & 0.15 & 0.93 & $0.88-0.99$ & 0.03 \\
\hline SOFA & 1.08 & $0.98-1.20$ & 0.12 & 1.14 & $1.05-1.25$ & 0.004 \\
\hline Bicarbonate_Ohr & 0.87 & $0.83-0.91$ & $<0.001$ & 1.03 & $0.99-1.08$ & 0.13 \\
\hline Albumin_Ohr & 2.01 & $1.01-3.99$ & 0.05 & 0.66 & $0.40-1.10$ & 0.11 \\
\hline
\end{tabular}

We categorized patients into three groups according to the changes in phosphorus and RDW-CV levels during 48 hours after CRRT initiation using the median values of the changes, $-2.0 \mathrm{mg} / \mathrm{dL}$ and $-0.2 \%$, respectively. We chose the lowest mortality group as the reference group. Delta phosphorus groups were categorized as increased (delta phosphorus $>0$ ), stable (delta phosphorus between median to 0), and decreased (delta phosphorus $<$ median). Delta RDW-CV groups were categorized as increased (delta RDW-CV > 0), stable (delta RDW-CV between median to 0), and decreased (delta RDW-CV < median). Phoxilium (-): the group of patients who did not receive Phoxilium (Baxter Healthcare Ltd., Norfolk, UK) within 48 hours of CRRT initiation. Phoxilium (+): the group of patients who received Phoxilium within 48 hours of CRRT initiation.

$\mathrm{BMI}$, body mass index; $\mathrm{Cl}$, confidence interval; CRRT, continuous renal replacement therapy; HR, hazard ratio; RDW-CV, red cell distribution width-coefficient of variation; SOFA, Sequential Organ Failure Assessment.

was not significantly different between the two groups, the mean SOFA_0hr score in the P group was higher than that in the NP group ( $8.7 \pm 3.4$ in the NP group vs. $9.4 \pm 3.1$ in the $\mathrm{P}$ group, $\mathrm{p}=0.08$ ). These results indicated that the $\mathrm{P}$ group had more severe inflammation and malnutrition status, leading to a more severe disease course than the NP group. After 48 hours of CRRT, SOFA_48hr was higher in the P group than in the NP group, which indicated a higher disease severity.

Hyperphosphatemia is commonly observed in patients with AKI due to decreased renal excretion. Additionally, it is associated with high mortality because it indicates disease severity and direct phosphorus toxicity. Within 2 to 3 days of CRRT, serum phosphorus levels mostly return to the normal range via extracorporeal clearance [21]. Thus, if hy- 

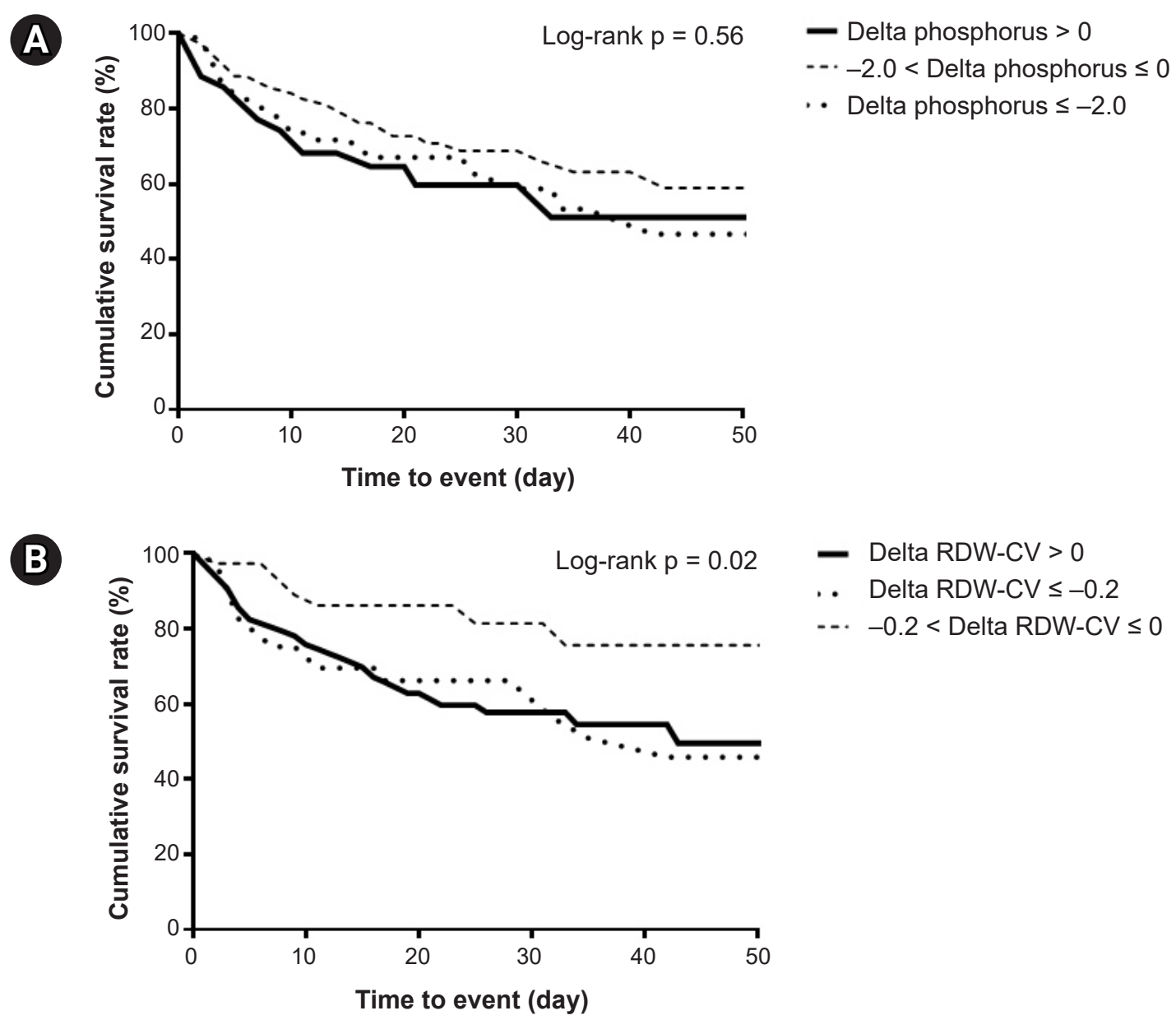

Figure 3. Kaplan-Meier plots for all-cause mortality according to changes in phosphorus and RDW-CV levels during 48 hours of CRRT in the Phoxilium group. We categorized patients into three groups according to the changes in phosphorus and RDW-CV levels between 0 hour and 48 hours: increased, stable, and decreased phosphorus and RDW-CV levels. We divided stable and decreased phosphorus and RDW-CV groups using the median value of the changes, $-2.0 \mathrm{mg} / \mathrm{dL}$ and $-0.2 \%$, respectively. In the Phoxilium group, the Kaplan-Meier curve for all-cause mortality showed that time to death was not different in patients with changes in phosphorus (Fig. $3 \mathrm{~A}, \mathrm{p}=0.56$ ), but it was significantly different in patients with changes in RDW-CV levels (Fig. 3B, $p=0.02$ ).

CRRT, continuous renal replacement therapy; delta phosphorus, changes in phosphorus during 48 hours of CRRT; delta RDW-CV, changes in RDW-CV during 48 hours of CRRT; RDW-CV, red cell distribution width-coefficient of variation. Phoxilium: Baxter Healthcare Ltd., Norfolk, UK.

perphosphatemia is not corrected even after CRRT, the allcause mortality risk is expected to increase. In the NP group, the increased phosphorus levels, compared with the stable or decreased phosphorus levels after 48 hours of CRRT, showed significantly higher all-cause mortality risks. In the P group, the increased phosphorus levels after 48 hours of CRRT showed increased all-cause mortality. Receiver operating characteristic curve analyses of phosphorus_0hr, phosphorus_48hr, and delta phosphorus in the NP and P group showed that the area under the curve (AUC) was largest for delta phosphorus of the NP group (AUC, 0.774).

Phosphorus plays an important role in all body functions, especially in nerve and muscle functions. If hypophosphatemia occurs in various conditions, myocardial contraction and granulocyte phagocytic activity decrease, and the development of arrhythmia increases [22-24]. It has also been associated with prolonged mechanical ventilation in critically ill patients with AKI $[3,25]$. In several previous studies, most of the harmful effects caused by hypophosphatemia occurred in patients with very severe phosphorus deficien- 


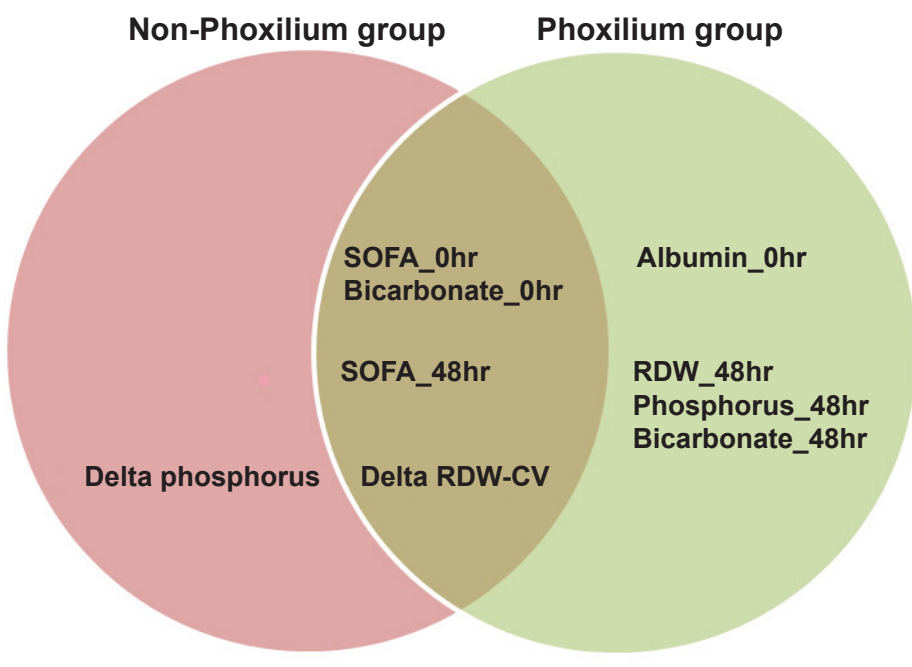

Figure 4. Diagram showing risk factors for all-cause mortality in non-Phoxilium and Phoxilium groups.

CRRT, continuous renal replacement therapy; delta phosphorus, changes in phosphorus during 48 hours of CRRT; delta RDW-CV, changes in RDW-CV during 48 hours of CRRT; RDW-CV, red cell distribution width-coefficient of variation; SOFA, Sequential Organ Failure Assessment. Phoxilium: Baxter Healthcare Ltd., Norfolk, UK.

cies, defined as a level of $<1.0 \mathrm{mg} / \mathrm{dL}$. Although hypophosphatemia cases increased in number from 11 cases $(4.0 \%)$ to 32 cases (11.7\%) after 48 hours of CRRT in the present study, severe hypophosphatemia did not occur. Thus, in line with the results of previous studies, Phoxilium was effective in preventing hypophosphatemia in patients with AKI undergoing CRRT [15,26,27].

The RDW-CV level is easy to measure and is recognized as a marker of adverse outcomes. Inflammation and oxidative stress are thought to be major factors in the pathogenesis underlying the association between RDW-CV and all-cause mortality [28]. Additionally, high RDW-CV levels were associated with declining estimated glomerular filtration levels, irrespective of anemia [28]. In some cases, pre-treatment high RDW-CV levels were related to prognosis, but in other studies, the dynamic change was related to adverse outcomes in patients with sepsis [11,29]. In this study, initial RDW-CV was not a significant risk factor for allcause mortality in the NP and P groups. However, a higher dynamic change in RDW-CV was associated with a higher all-cause mortality rate. In the P group, a decreased level of delta RDW-CV was a risk factor for all-cause mortality. In the comparison of the mean values of RDW-CV_Ohr and RDWCV_48hr in each delta RDW-CV group, both values in the decreased group were significantly higher than those in the stable and increased groups (data not shown). Therefore, in agreement with the result that higher RDW-CV_48hr is a risk factor for all-cause mortality, the increased and decreased delta RDW-CV groups showed increased risks of all-cause mortality compared to the stable group.

In this study, initial bicarbonate levels did not differ between the NP and P groups $(16.2 \pm 6.6 \mathrm{mEq} / \mathrm{L}$ in the NP group vs. $16.7 \pm 6.1 \mathrm{mEq} / \mathrm{L}$ in the $\mathrm{P}$ group, $\mathrm{p}>0.05$ ). After 48 hours of CRRT, bicarbonate levels were higher in the NP group, although the mean values of both groups were within the normal range $(23.1 \pm 5.5 \mathrm{mEq} / \mathrm{L}$ in the NP group vs. $21.7 \pm 4.0$ $\mathrm{mEq} / \mathrm{L}$ in the $\mathrm{P}$ group, $\mathrm{p}=0.03$ ). The lower bicarbonate levels in Phoxilium than in the other solutions ( $30 \mathrm{mmol} / \mathrm{L}$ in Phoxilium vs. $32 \mathrm{mmol} / \mathrm{L}$ in Hemosol, PrismaSol 2, and PrismaSol 4) might have resulted in relatively reduced serum bicarbonate levels [15]. Higher bicarbonate_0hr was associated with increased all-cause mortality in both groups, with lower bicarbonate_48hr being associated with mortality only in the $\mathrm{P}$ group. Patients with AKI commonly have metabolic acidosis, which is an independent predictor of unfavorable outcomes [30-32]. However, as is well known, the serum bicarbonate level itself cannot reflect the exact acid-base balance status, and compensatory mechanisms should be considered when interpreting blood gas analysis. Thus, we conducted further analyses of ventilator use and blood gas analysis results in 
the survivor and non-survivor groups, as shown in Supplementary Table 5 (available online). The non-survivor group showed a higher percentage of ventilator use and $\mathrm{pCO}_{2}{ }_{2} \mathrm{hr}$ ( $p<0.001$ and $\mathrm{p}=0.002$, respectively) with higher bicarbonate_0hr $(\mathrm{p}<0.001)$ than the survivor group. Furthermore, the additional univariable analysis of Cox regression showed that higher $\mathrm{pCO}_{2-} 0 \mathrm{hr}$ (HR, 1.04; 95\% CI, 1.01-1.06; $\mathrm{p}=0.004$ ) and bicarbonate_0hr (HR, 1.08; 95\% CI, 1.02-1.15; p = 0.007) were risk factors for all-cause mortality.

When interpreting the results above, the combination of metabolic and respiratory acidemia seemed to be the mainstay of blood gas status in the non-survivor group, whereby primary metabolic acidemia was prominent in the survivor group. Patients with combined acidemia showed higher mortality than those with either metabolic or respiratory acidemia alone in a critically ill state [33]. Therefore, patients showing a mixed disorder of acid-base balance, also referred to as having higher $\mathrm{pCO}_{2-} \mathrm{Ohr}$ and bicarbonate_0hr in this study, were associated with adverse outcomes. After 48 hours of CRRT, more severe metabolic acidosis with lower bicarbonate levels in the P group was related to adverse outcomes.

Hypoalbuminemia is associated with several pathological conditions, such as nutritional deficiency and chronic inflammation. It is a predictive marker of short- and longterm mortality in patients undergoing CRRT [10]. This study also showed that low initial albumin levels indicated adverse outcomes in the NP and P groups. However, after fully adjusting for confounding factors, hypoalbuminemia was significant only in the $\mathrm{P}$ group. This difference seems to be due to the different levels of initial albumin between the groups. Initial albumin levels were higher in the NP group than in the P group $(3.5 \pm 0.9 \mathrm{~g} / \mathrm{dL}$ in the NP group vs. $3.1 \pm 0.6 \mathrm{~g} / \mathrm{dL}$ in the $\mathrm{P}$ group, $\mathrm{p}<0.001$ ). As phosphorus reflects nutritional status, basal nutritional status was expected to be poor in the P group.

This study had several limitations. Although the analysis was performed with appropriate adjustments, the possibility of residual confounders due to the retrospective nature of the study cannot be excluded. Additionally, most of the patients were Korean; thus, there is a limitation in applying the findings to other races. Compared with Hemosol-B0, Phoxilium contributes to relative hypocalcemia and metabolic acidosis [15]. Although there are differences in the compositions of Phoxilium and other dialysate fluids, the changes in corrected calcium and bicarbonate levels were not significantly different between the NP and P groups. As this is a retrospective analysis, efforts to correct hypocalcemia and metabolic acidosis with intravenous or oral calcium and bicarbonate supplementation were not controlled. Therefore, calcium_ $48 \mathrm{hr}$ and bicarbonate_48hr might not reflect solution differences.

In conclusion, this study strongly suggests that in patients with AKI undergoing CVVHDF, the difference in disease severity and risk factors for all-cause mortality is affected by the initial phosphorus levels and the use of Phoxilium. Additionally, the effects of changes in phosphorus and RDW-CV levels by short-term CVVHDF using different compositions of dialysate fluids on all-cause mortality differed according to the initial phosphorus level.

\section{Conflicts of interest}

All authors have no conflicts of interest to declare.

\section{Funding}

This work was supported by a clinical research grant from Pusan National University Hospital in 2020.

\section{Authors' contributions}

Conceptualization: SHS, HJK, DWK

Formal analysis: DWK, HJK, JMK

Funding acquisition: SHS

Investigation: MH, YHJ, EYS

Project administration: SSH

Writing-original draft: DWK, HJK

Writing-review \& editing: DWK, HJK, SHS All authors read and approved the final manuscript.

\section{ORCID}

Da Woon Kim, https://orcid.org/0000-0002-9471-5976

Hyo Jin Kim, https://orcid.org/0000-0001-9289-9073

Jin Mi Kim, https://orcid.org/0000-0002-6715-0447

You Hyun Jeon, https://orcid.org/0000-0001-7318-5753

Miyeun Han, https://orcid.org/0000-0001-7304-2496

Eun Young Seong, https://orcid.org/0000-0002-6006-0051

Sang Heon Song, https://orcid.org/0000-0002-8218-6974 


\section{References}

1. Santiago MJ, López-Herce J, Urbano J, et al. Complications of continuous renal replacement therapy in critically ill children: a prospective observational evaluation study. Crit Care 2009;13:R184.

2. Sharma S, Brugnara C, Betensky RA, Waikar SS. Reductions in red blood cell 2,3-diphosphoglycerate concentration during continuous renal replacment therapy. Clin J Am Soc Nephrol 2015; 10:74-79.

3. Lim C, Tan HK, Kaushik M. Hypophosphatemia in critically ill patients with acute kidney injury treated with hemodialysis is associated with adverse events. Clin Kidney J 2017;10:341-347.

4. Jung SY, Kwon J, Park S, et al. Phosphate is a potential biomarker of disease severity and predicts adverse outcomes in acute kidney injury patients undergoing continuous renal replacement therapy. PLoS One 2018;13:e191290.

5. Ratanarat R, Brendolan A, Volker G, et al. Phosphate kinetics during different dialysis modalities. Blood Purif 2005;23:83-90.

6. Song YH, Seo EH, Yoo YS, Jo YI. Phosphate supplementation for hypophosphatemia during continuous renal replacement therapy in adults. Ren Fail 2019;41:72-79.

7. Santiago MJ, López-Herce J, Muñoz R, et al. Stability of continuous renal replacement therapy solutions after phosphate addition: an experimental study. Ther Apher Dial 2011;15:75-80.

8. Troyanov S, Geadah D, Ghannoum M, Cardinal J, Leblanc M. Phosphate addition to hemodiafiltration solutions during continuous renal replacement therapy. Intensive Care Med 2004;30:1662-1665.

9. Oh HJ, Park JT, Kim JK, et al. Red blood cell distribution width is an independent predictor of mortality in acute kidney injury patients treated with continuous renal replacement therapy. Nephrol Dial Transplant 2012;27:589-594.

10. Moon JJ, Kim Y, Kim DK, Joo KW, Kim YS, Han SS. Association of hypoalbuminemia with short-term and long-term mortality in patients undergoing continuous renal replacement therapy. Kidney Res Clin Pract 2020;39:47-53.

11. Ju XF, Wang F, Wang L, et al. Dynamic change of red cell distribution width levels in prediction of hospital mortality in Chinese elderly patients with septic shock. Chin Med J (Engl) 2017;130:1189-1195.

12. Farrow EG, Yu X, Summers LJ, et al. Iron deficiency drives an autosomal dominant hypophosphatemic rickets (ADHR) phenotype in fibroblast growth factor-23 (Fgf23) knock-in mice. Proc Natl Acad Sci U S A 2011;108:E1146-E1155.
13. Wolf M, Koch TA, Bregman DB. Effects of iron deficiency anemia and its treatment on fibroblast growth factor 23 and phosphate homeostasis in women. J Bone Miner Res 2013;28:1793-1803.

14. van Breda F, Emans ME, van der Putten K, et al. Relation between red cell distribution width and fibroblast growth factor 23 cleaving in patients with chronic kidney disease and heart failure. PLoS One 2015;10:e128994.

15. Chua HR, Schneider AG, Baldwin I, Collins A, Ho L, Bellomo R. Phoxilium vs Hemosol-B0 for continuous renal replacement therapy in acute kidney injury. J Crit Care 2013;28:884 .

16. Godinjak A, Iglica A, Rama A, et al. Predictive value of SAPS II and APACHE II scoring systems for patient outcome in a medical intensive care unit. Acta Med Acad 2016;45:97-103.

17. Park S, Lee S, Jo HA, et al. Epidemiology of continuous renal replacement therapy in Korea: Results from the National Health Insurance Service claims database from 2005 to 2016. Kidney Res Clin Pract 2018;37:119-129.

18. Wald R, McArthur E, Adhikari NK, et al. Changing incidence and outcomes following dialysis-requiring acute kidney injury among critically ill adults: a population-based cohort study. Am J Kidney Dis 2015;65:870-877.

19. Carlson N, Hommel K, Olesen JB, et al. Dialysis-requiring acute kidney injury in Denmark 2000-2012: time trends of incidence and prevalence of risk factors-a nationwide study. PLoS One 2016;11:e0148809.

20. Akhoundi A, Singh B, Vela M, et al. Incidence of adverse events during continuous renal replacement therapy. Blood Purif 2015;39:333-339.

21. Tan HK, Bellomo R, M'Pis DA, Ronco C. Phosphatemic control during acute renal failure: intermittent hemodialysis versus continuous hemodiafiltration. Int J Artif Organs 2001;24:186-191.

22. Craddock PR, Yawata Y, VanSanten L, Gilberstadt S, Silvis S, Jacob HS. Acquired phagocyte dysfunction. A complication of the hypophosphatemia of parenteral hyperalimentation. N Engl J Med 1974;290:1403-1407.

23. Saglikes Y, Massry SG, Iseki K, et al. Effect of phosphate depletion on blood pressure and vascular reactivity to norepinephrine and angiotensin II in the rat. Am J Physiol 1985;248(1 Pt 2):F93-F99.

24. Davis SV, Olichwier KK, Chakko SC. Reversible depression of myocardial performance in hypophosphatemia. Am J Med Sci 1988;295:183-187.

25. Alsumrain MH, Jawad SA, Imran NB, Riar S, DeBari VA, Adelman M. Association of hypophosphatemia with failure-to-wean from mechanical ventilation. Ann Clin Lab Sci 2010;40:144-148.

26. Broman M, Carlsson O, Friberg H, Wieslander A, Godaly G. 
Phosphate-containing dialysis solution prevents hypophosphatemia during continuous renal replacement therapy. Acta Anaesthesiol Scand 2011;55:39-45.

27. Chua HR, Baldwin I, Ho L, Collins A, Allsep H, Bellomo R. Biochemical effects of phosphate-containing replacement fluid for continuous venovenous hemofiltration. Blood Purif 2012;34:306-312.

28. Lippi G, Targher G, Montagnana M, Salvagno GL, Zoppini G, Guidi GC. Relationship between red blood cell distribution width and kidney function tests in a large cohort of unselected outpatients. Scand J Clin Lab Invest 2008;68:745-748.

29. Hu ZD, Lippi G, Montagnana M. Diagnostic and prognostic value of red blood cell distribution width in sepsis: a narrative review. Clin Biochem 2020;77:1-6.
30. Bailey JL, Mitch WE. The implications of metabolic acidosis in intensive care unit patients. Nephrol Dial Transplant 1998; 13:837-839.

31. Clermont G, Acker CG, Angus DC, Sirio CA, Pinsky MR, Johnson JP. Renal failure in the ICU: comparison of the impact of acute renal failure and end-stage renal disease on ICU outcomes. Kidney Int 2002;62:986-996.

32. Lee SW, Hong YS, Park DW, et al. Lactic acidosis not hyperlactatemia as a predictor of in hospital mortality in septic emergency patients. Emerg Med J 2008;25:659-665.

33. Mochizuki K, Fujii T, Paul E, et al. Acidemia subtypes in critically ill patients: An international cohort study. J Crit Care 2021;64:1017. 